\title{
EDITORIAL
}

\section{Fehlurteile im Strafverfahren}

\author{
Hans-Ludwig Kröber · Dieter Dölling
}

Online publiziert: 8. Oktober 2013

(C) Springer-Verlag Berlin Heidelberg 2013

Das Schwerpunktthema des vorliegenden Hefts der Zeitschrift Forensische Psychiatrie, Psychologie, Kriminologie sind Fehlurteile im Strafverfahren. Das Bundesverfassungsgericht hat in seiner grundlegenden Entscheidung zur strafprozessualen Verständigung - Urteil vom 19.03.2013, Az.2 BvR 2628/10 u. a. - die verfassungsrechtliche Pflicht des Staates zur Wahrheitsermittlung im Strafverfahren betont. Durch Fehlurteile wird die Erfüllung dieser Pflicht verfehlt. Falsche Verurteilungen können verheerende Auswirkungen auf den zu Unrecht verurteilten Angeklagten haben, und auch ein fehlerhafter Freispruch kann das Rechtsbewusstsein massiv beeinträchtigen. Gleichwohl ist die Problematik des Fehlurteils in Deutschland erst in jüngster Zeit durch das Bekanntwerden einiger fehlerhafter Urteile stärker in das Bewusstsein der Öffentlichkeit gelangt. Diese Urteile zeigen auch, dass die Problematik dringend einer intensiveren wissenschaftlichen Behandlung bedarf. Im vorliegenden Heft setzen sich 5 Beiträge mit der Problematik auseinander. Jörg-Martin Jehle erörtert den Begriff des Fehlurteils, stellt ausländische Befunde zur Fehlurteilsproblematik dar und befasst sich mit Anhaltspunkten für das Ausmaß von Fehlurteilen in der deutschen Strafrechtspflege. Ein Grund für Fehlurteile können falsche Geständnisse sein. Renate

Prof. Dr. H.-L. Kröber ( $₫)$

Institut für Forensische Psychiatrie,

Charité - Universitätsmedizin Berlin,

Oranienburger Str. 285, 13437 Berlin, Deutschland

E-Mail: Hans-Ludwig.Kroeber@charite.de

Prof. Dr. D. Dölling

Institut für Kriminologie, Juristische Fakultät,

Ruprecht-Karls-Universität, Friedrich-Ebert-Anlage 6-10, 69117 Heidelberg, Deutschland

E-Mail: doelling@krimi.uni-heidelberg.de
Volbert erörtert in ihrem Beitrag, bei welchen Personengruppen ein besonderes Risiko besteht, ein falsches Geständnis abzulegen, welche Vernehmungsstrategien die Gefahr eines falschen Geständnisses erhöhen, welche Rolle Voreinstellungen der Befragenden spielen und welche Möglichkeiten bestehen, falsche Geständnisse zu erkennen. Hans-Ludwig Kröber schildert anhand von 3 Fällen die Entstehung von Falschbeschuldigungen. Wolfgang Pfister legt die Risiken für ein Fehlurteil dar, die mit einem „Deal“ im Strafverfahren verbunden sind, und zeigt, dass diesen Gefahren durch die sorgfältige Beachtung der gesetzlichen Regelungen über die Verständigung begegnet werden kann. Aus der Sicht eines Strafverteidigers schildert Johann Schwenn typische Merkmale von Fehlurteilen, die insbesondere auf Falschbeschuldigungen beruhen.

Außerdem enthält dieses Heft 4 weitere Beiträge. Reinhard Eher et al. berichten über die Validierung der Treffsicherheit der Prognoseverfahren Static-99 und Stable-2007 bei entlassenen österreichischen Maßregelpatienten. Johannes Koranyi und Torsten Verrel erörtern die Auswirkungen einer Entscheidung des Europäischen Gerichtshofs für Menschenrechte über die Zulässigkeit ärztlicher Suizidbeihilfe. Mit der Abgrenzung von Autismus und „psychopathy“ befassen sich Jens Roberz, Gerd Lehmkuhl und Kathrin Sevecke anhand einer Literaturübersicht und einer ausführlichen Fallgeschichte. Christian Hartl untersucht die Glaubhaftigkeit der Angaben von ehemaligen Maßregelvollzugspatienten durch einen Vergleich mit den Angaben von Bewährungshelfern und Auszügen aus dem Bundeszentralregister. 